\title{
INVERSE $H$-SEMIGROUPS AND $t$-SEMISIMPLE INVERSE $H$-SEMIGROUPS $\left({ }^{1}\right)$
}

\author{
BY \\ MARY JOEL JORDAN, S.C.
}

\begin{abstract}
An $H$-semigroup is a semigroup such that both its right and left congruences are two-sided. A semigroup is $t$-semisimple provided the intersection of all its maximal modular congruences is the identity relation. We prove that a semigroup is an inverse $H$-semigroup if and only if it is a semilattice of disjoint Hamiltonian groups. Using the set $E$ of idempotents of $S$ as the semilattice, we show that an inverse $H$-semigroup $S$ is $t$-semisimple if and only if for each pair of groups $G_{e}, G_{f}$ in the semilattice, with $f \geqq e$ in $E$, the homomorphism $\varphi_{f, e}$ on $G_{f}$ into $G_{e}$, defined by $a \varphi_{f, e}=a e$, is a monomorphism; and for each $e$ in $E$, for each $a \neq e$ in $G_{e}$, there exists a subsemigroup $T_{p}$ of $S$ such that $a \notin T_{p}$ and, for each $f$ in $E, T_{p} \cap G_{f}=H_{f}$, where $H_{f}=G_{f}$ or $H_{f}$ is a maximal subgroup of prime index $p$ in $G_{f}$.
\end{abstract}

Introduction. In this paper we adopt the definition of a Hamiltonian semigroup presented by R. H. Oehmke [6]. Let $\sigma$ be an equivalence relation on a semigroup $S$. If $a$ is equivalent to $b$ we shall write $a \sigma b$. The $\sigma$-class containing $a$ will be denoted by $\sigma_{a}$. An equivalence relation $\sigma$ on a semigroup $S$ is a right (left) congruence provided $a, b, c \in S$ and $a \sigma b$ imply $(a c) \sigma(b c)((c a) \sigma(c b))$. If an equivalence relation is both a right and a left congruence, we shall call it a two-sided congruence or, more briefly, a congruence. We use the natural partial ordering on relations and say that $\sigma \leqq \rho$ if and only if $a, b \in S$ and $a \sigma b$ imply $a \rho b$. Clearly, the identity relation $\iota$ and the universal relation $\nu$ are congruences and $\iota \leqq \sigma \leqq \nu$ for each congruence $\sigma$ on $S$. A congruence $\sigma \neq \nu$ is called maximal if for each congruence $\sigma^{\prime}$ on $S$ such that $\sigma \leqq \sigma^{\prime} \leqq \nu$, either $\sigma=\sigma^{\prime}$ or $\sigma^{\prime}=\nu$. An $H$-semigroup $S$ is defined to be a semigroup such that every right congruence and every left congruence is a twosided congruence on $S$. Since a subgroup of a group is normal if and only if its corresponding right (left) congruence is two-sided, then the class of $H$-semigroups contains the Hamiltonian groups in addition to the commutative semigroups, where we include all commutative groups in the set of all Hamiltonian groups. An inverse $H$-semigroup is a semigroup that is an inverse semigroup as well as an $H$-semigroup.

Received by the editors July 16, 1970.

AMS 1969 subject classifications. Primary 2092, 2093; Secondary 2058.

Key words and phrases. Semigroup, inverse semigroup, $H$-semigroup, $t$-semisimple semigroup, Hamiltonian group, semilattice, maximal congruence, modular congruence.

( $\left.{ }^{1}\right)$ These results are part of the author's doctoral dissertation written at the University of Iowa under the supervision of Professor Robert H. Oehmke. This research was partially supported by an NSF Science Faculty Fellowship and supported in part under ONR Contract N0014-68-A-0500. 
Using the above definitions we prove in $\$ 2$ that a semigroup is an inverse $H$ semigroup if and only if it is a semilattice of disjoint Hamiltonian groups.

We define $\tau$ to be the intersection of all the maximal modular congruences on a semigroup $S$, where a congruence $\sigma$ is called modular if there is an element $e$ of $S$ such that (ea) $\sigma a$ and ( $a e) \sigma a$ for all $a$ in $S$. The element $e$ is called an identity for $\sigma$. We refer to $\tau$ as the $t$-radical of $S$. $S$ is said to be $t$-semisimple if $\tau=\iota$ [7]. In $\S 3$, we give necessary and sufficient conditions for an inverse $H$-semigroup $S$ to be $t$-semisimple. This result has several nontrivial corollaries.

The author expresses her gratitude to Professor Robert H. Oehmke for his guidance in the preparation of this paper.

1. Preliminary definitions and results. An element $b$ of a semigroup $S$ is an inverse of an element $a$ of $S$ provided $a b a=a$ and $b a b=b$. $S$ is an inverse semigroup provided every element of $S$ has a unique inverse. The inverse of an element $a$ of an inverse semigroup $S$ will be denoted by $a^{-1}$ so that $a a^{-1} a=a$ and $a^{-1} a a^{-1}=a^{-1}$. The preceding definitions are taken from [1].

We also make use of the following results which have been proved in [1, pp. 2330]. Let $S$ be an inverse semigroup. The set $E$ of idempotents $S$ is a semilattice, i.e., a commutative idempotent semigroup with the induced ordering $e \leqq f$ if and only if $e f=e$. If $a, b \in S$ then $\left(a^{-1}\right)^{-1}=a$ and $(a b)^{-1}=b^{-1} a^{-1}$. Every principal right ideal and every principal left ideal of $S$ has a unique idempotent generator. The idempotent $e=a a^{-1}\left(f=a^{-1} a\right)$ is the unique idempotent generator of $a S(S a)$.

A left (right) zero of a semigroup $S$ is an element $a$ of $S$ such that $a s=a(s a=a)$, for each $s \in S$ [1]. Let $c$ be a left (right) zero of an inverse semigroup $S$. Then for each $s \in S, c s c=c$ implies $s c s=s c(s c s=c s)$ is an inverse of $c$. But $c$ has a unique inverse, namely $c$, so that, for each $s \in S, s c=c(c s=c)$. Hence $c$ is a right (left) zero of $S$ and $S$ has at most one (left, right) zero.

In [6] Oehmke proved the following result which we state as a lemma.

LEMMA 1.1. If $S$ is an H-semigroup and $I$ is a right (left) ideal of $S$ then, for any $b$ in $S, b I \subseteq I(I b \subseteq I)$ or $b I=\{c\}$ where $c$ is a left zero $(I b=\{c\}$ where $c$ is a right zero).

We use this result to show that every one-sided ideal of an inverse $H$-semigroup $S$ is two-sided and thus we obtain that $S$ is a semilattice of disjoint groups.

\section{Lemma 1.2. A right (left) ideal of an inverse $H$-semigroup $S$ is two-sided.}

Proof. Let $I$ be a right ideal of $S$ and $b \in S$. By Lemma 1.1, either $b I \subseteq I$ or $b I=\{c\}$ where $c$ is a left zero. If the latter is true, then, since $c$ is also a right zero and $I$ is a right ideal, we have $\{c\}=I c \subseteq I$ so that $b I=\{c\} \subseteq I$ and $I$ is a left ideal in either case. By a similar proof, any left ideal of $S$ is a right ideal of $S$.

Let $S$ be an inverse $H$-semigroup and $e$ an idempotent of $S$. Since $S e$ is an ideal and $e \in S e$, it follows that $S e=e S$. Then for any $a \in S$ we have $a e=a$ if and only if $e a=a$. But for $a \in S$ there exists a unique element $a^{-1} \in S$ such that $a a^{-1}$ and 
$a^{-1} a$ are idempotents. Thus we have $\left(a a^{-1}\right) a=a$ so that $a\left(a a^{-1}\right)=a$, and also $a=a\left(a^{-1} a\right)$ so that $\left(a^{-1} a\right) a=a$. Hence

$$
a^{-1} a=a^{-1}\left(a a a^{-1}\right)=\left(a^{-1} a a\right) a^{-1}=a a^{-1} .
$$

It is well known that if every element of an inverse semigroup $S$ commutes with its inverse then $S$ is a union of disjoint groups. Thus we have the following lemma:

LemMA 1.3. If $S$ is an inverse $H$-semigroup then $S$ is a union of disjoint groups.

If $Y$ is a semilattice such that $S=\bigcup\left\{S_{\alpha}: \alpha \in Y\right\}$ is a decomposition of $S$ such that, for every pair of elements $\alpha, \beta$ of $Y$, there is an element $\gamma$ of $Y$ such that $S_{\alpha} S_{\beta} \subseteq S_{\gamma}$, we say that $S$ is the union of the semilattice $Y$ of semigroups $S_{\alpha}, \alpha \in Y$. We also abbreviate the expression and say that $S$ is a semilattice of semigroups of type $\mathscr{C}$ to mean that $S$ is the union of the semilattice of semigroups $S_{\alpha}, \alpha \in Y$, where each $S_{\alpha}$ is of type $\mathscr{C}$.

Let $S$ be an inverse $H$-semigroup and let $G_{e}=\left\{b \in S: b b^{-1}=e\right\}$. It readily follows that $G_{e}$ is a maximal subgroup of $S$ and $S=\bigcup\left\{G_{e}: e \in E\right\}$, where $G_{e} \cap G_{f}=\varnothing$ for $e \neq f$. Using Lemma 1.3, we obtain the result that $E$ is contained in the center of $S[1$, pp. 127-128] so that Theorem 1 follows.

THEOREM 1. If $S$ is an inverse $H$-semigroup then $S$ is a semilattice of disjoint groups, and if $f \geqq e$ in $E$, the mapping $\varphi_{f, e}$, defined by $a \varphi_{f, e}=$ ae where $a \in G_{f}$, is a homomorphism of $G_{f}$ into $G_{e}$. Also, $\varphi_{f, f}$ is the identity mapping of $G_{f}$ and iff $\geqq e \geqq g$, then $\varphi_{f, e} \varphi_{e, g}=\varphi_{f, g}$. Moreover, every product in $S$ is known, since for $a \in G_{f}$ and $b \in G_{e}, a b=\left(a \varphi_{f, f e}\right)\left(b \varphi_{e, f e}\right)$.

2. In this section we shall obtain a characterization of inverse $H$-semigroups, namely:

THEOREM 2. A semigroup $S$ is an inverse $H$-semigroup if and only if $S$ is a semilattice of disjoint Hamiltonian groups.

Proof. Let $\delta$ be a right congruence on an inverse $H$-semigroup $S$. Let $G_{e}$ be a maximal subgroup of $S$ and let $\delta^{\prime}$ be the restriction of $\delta$ to $G_{e}$. By a straightforward argument, it can be shown that there is a subgroup $H_{e}$ of $G_{e}$ such that $\delta^{\prime}$ is the right congruence on $G_{e}$ induced by $H_{e}$. On the other hand, for any $e$ in $E$, let $H_{e}$ be any subgroup of $G_{e}$. Let $\sigma$ be the right congruence induced by $H_{e}$ on $G_{e}$. If $f<e$, let $H_{f}=G_{f}$. If $e$ and $f$ are not comparable, written $e$ ? $f$, let $H_{f}=G_{f}$. If $f \geqq e$, let $H_{f}=\left(H_{e}\right) \varphi_{f, e}$ where $\varphi_{f, e}$ is the homomorphism on $G_{f}$ into $G_{e}$. Let $a, b \in S$. Write

$$
a \sigma^{\prime} b \Leftrightarrow a, b \in G_{f} \text { and } a b^{-1} \in H_{f} \quad \text { for some } f \in E .
$$

It readily follows that $\sigma^{\prime}$ is an equivalence relation on $S$. Assume $a \sigma^{\prime} b$ and let $c \in G_{k}$. If (1) $k<e, f<e$ or (2) $k<e, f$ ? $e$ then either $f k<e$ or $f k$ ? $e$ so that $H_{f k}=G_{f k}$. In these cases $H_{f}=G_{f}$ and $H_{k}=G_{k}$. Hence $H_{f} \cdot H_{k} \subseteq H_{f k}$. A similar argument obtains the same result in each of the remaining cases so that $(a c) \sigma^{\prime}(b c)$ and $\sigma^{\prime}$ 
is a (right) congruence on $S$. Therefore, if $a, b \in G_{e}$ and $a \sigma b$, then $a b^{-1} \in H_{e}$ implies $\sigma$ is the restriction of $\sigma^{\prime}$ to $G_{e}$ so that $\sigma$ is a congruence on $G_{e}$. Then $H_{e}$ is a normal subgroup of $G_{e}$ and $G_{e}$ is Hamiltonian. Hence, if $S$ is an inverse $H$-semigroup, then $S$ is a semilattice of disjoint Hamiltonian groups.

Let $Y$ be any semilattice and to each element $\alpha$ of $Y$ assign a group $G_{\alpha}$ such that $G_{\alpha}$ and $G_{\beta}$ are disjoint if $\alpha \neq \beta$ in $Y$. To each pair of elements $\alpha, \beta$ of $Y$ such that $\alpha>\beta$, assign a homomorphism $\varphi_{\alpha, \beta}$ of $G_{\alpha}$ into $G_{\beta}$ such that if $\alpha>\beta>\gamma$, then $\varphi_{\alpha, \beta} \varphi_{\beta, \gamma}=\varphi_{\alpha, \gamma}$. Let $\varphi_{\alpha, \alpha}$ be the identity automorphism of $G_{\alpha}$. Let $S$ be the union of all the groups $G_{\alpha}, \alpha \in Y$, and define the product of any two elements $a_{\alpha}, b_{\beta}$ of $S$ $\left(a_{\alpha}\right.$ in $G_{\alpha}, b_{\beta}$ in $\left.G_{\beta}\right)$ by $a_{\alpha} b_{\beta}=\left(a_{\alpha} \varphi_{\alpha, \alpha \beta}\right)\left(b_{\beta} \varphi_{\beta, \alpha \beta}\right)$. Then $S$ is an inverse semigroup which is a union of groups [1, p. 128].

Assume the groups $G_{\alpha}, \alpha \in Y$, are Hamiltonian. It remains to show that $S$ is an $H$-semigroup. Let $\sigma$ be a right congruence on $S$. For each $G_{\alpha}, \sigma$ restricted to $G_{\alpha}$ induces a right congruence $\sigma_{\alpha}$ on $G_{\alpha}$. Since $G_{\alpha}$ is Hamiltonian then $\sigma_{\alpha}$ is two-sided so that $\sigma$ determines a normal subgroup $H_{\alpha}$ of $G_{\alpha}$. Let $e_{\alpha}$ be the identity of $G_{\alpha}$. Recall that $E$ is in the center of $S$ where $E$ is the semilattice of idempotents of $S$. Then we have

Further,

$$
\begin{aligned}
a_{\alpha} \sigma b_{\beta} & \Rightarrow\left(a_{\alpha} e_{\alpha} e_{\beta}\right) \sigma\left(b_{\beta} e_{\alpha} e_{\beta}\right) \Rightarrow\left(a_{\alpha} e_{\beta}\right) \sigma\left(b_{\beta} e_{\alpha}\right) \\
& \Rightarrow\left(a_{\alpha} e_{\beta}\right) \sigma_{\alpha \beta}\left(b_{\beta} e_{\alpha}\right) \Rightarrow a_{\alpha} e_{\beta}\left(b_{\beta} e_{\alpha}\right)^{-1}=a_{\alpha} b_{\beta}^{-1} \in H_{\alpha \beta}
\end{aligned}
$$

$$
a_{\alpha} \sigma b_{\beta} \Rightarrow\left(a_{\alpha} b_{\beta}^{-1}\right) \sigma e_{\beta} \Rightarrow\left(a_{\alpha} b_{\beta}^{-1}\right) \sigma e_{\alpha \beta}
$$

so that $e_{\alpha \beta} \sigma e_{\beta}$ and, by symmetry, $e_{\alpha \beta} \sigma e_{\alpha}$ so that $e_{\beta} \sigma e_{\alpha \beta} \sigma e_{\alpha}$.

Conversely,

$$
\begin{aligned}
a_{\alpha} b_{\beta}^{-1} \in H_{\alpha \beta} \text { and } e_{\beta} \sigma e_{\alpha \beta} \sigma e_{\alpha} & \Rightarrow\left(a_{\alpha} b_{\beta}^{-1}\right) \sigma e_{\alpha \beta} \sigma e_{\beta} \text { and } a_{\alpha} \sigma\left(a_{\alpha} e_{\beta}\right) \\
& \Rightarrow\left(a_{\alpha} e_{\beta}\right) \sigma b_{\beta} \text { and } a_{\alpha} \sigma b_{\beta} .
\end{aligned}
$$

Let $c_{\gamma} \in S$ and assume $a_{\alpha} \sigma b_{\beta}$.

$$
\begin{aligned}
a_{\alpha} \sigma b_{\beta} & \Rightarrow a_{\alpha} b_{\beta}^{-1} \in H_{\alpha \beta} \Rightarrow\left(a_{\alpha} b_{\beta}^{-1}\right) \sigma_{\alpha \beta} e_{\alpha \beta} \Rightarrow\left(a_{\alpha} b_{\beta}^{-1}\right) \sigma e_{\alpha \beta} \\
& \Rightarrow\left(a_{\alpha} b_{\beta}^{-1} e_{\gamma}\right) \sigma\left(e_{\alpha \beta} e_{\gamma}\right) \Rightarrow\left(a_{\alpha} b_{\beta}^{-1} e_{\gamma}\right) \sigma_{\alpha \beta \gamma} e_{\alpha \beta \gamma} \Rightarrow a_{\alpha} b_{\beta}^{-1} e_{\gamma} \in H_{\alpha \beta \gamma} .
\end{aligned}
$$

Since $H_{\alpha \beta \gamma}$ is a normal subgroup of $G_{\alpha \beta \gamma}$ then

and

$$
\left(c_{\gamma} e_{\alpha} e_{\beta}\right) a_{\alpha} b_{\beta}^{-1} e_{\gamma}\left(c_{\gamma} e_{\alpha} e_{\beta}\right)^{-1}=c_{\gamma} a_{\alpha} b_{\beta}^{-1} c_{\gamma}^{-1}=\left(c_{\gamma} a_{\alpha}\right)\left(c_{\gamma} b_{\beta}\right)^{-1} \in H_{\alpha \beta \gamma}
$$

$$
\left(c_{\gamma} a_{\alpha}\right)^{-1}\left(c_{\gamma} b_{\beta}\right) \in H_{\alpha \beta \gamma} .
$$

Further, $a_{\alpha} \sigma b_{\beta} \Rightarrow e_{\beta} \sigma e_{\alpha \beta} \sigma e_{\alpha} \Rightarrow e_{\gamma \alpha} \sigma e_{\gamma \alpha \beta} \sigma e_{\gamma \beta}$. Thus we have $\left(c_{\gamma} a_{\alpha}\right) \sigma\left(c_{\gamma} b_{\beta}\right)$ and $\sigma$ is also a left congruence. By an analogous proof, if $\sigma$ is a left congruence on $S$, then $\sigma$ is a right congruence on $S$. Hence $S$ is an inverse $H$-semigroup.

3. In this section we first identify the maximal modular congruences of an inverse $H$-semigroup $S$, and then obtain necessary and sufficient conditions for $S$ to be $t$-semisimple. 
A homomorphic image of an inverse semigroup is an inverse semigroup. Moreover, in any homomorphism, the inverse of an element is mapped onto the inverse of the image of that element [2, p. 57].

As one might also expect, the homomorphic image of an $H$-semigroup $S$ is an $H$-semigroup. For if $\psi$ is a homomorphism from $S$ onto $S^{\prime}, S^{\prime}$ is obviously a semigroup. And if $\mu^{\prime}$ is any right (left) congruence on $S^{\prime}$, we can define $\mu$ on $S$ by

$$
a \mu b \Leftrightarrow(a \psi) \mu^{\prime}(b \psi)
$$

Then $\mu$ is a congruence on $S$ and from this it follows that $\mu^{\prime}$ is a left (right) congruence on $S^{\prime}$.

Let $S$ be an $H$-semigroup. Let $I$ be an ideal of $S$ and $T$ a subsemigroup of $S$ such that $I \cup T=S$ and $I \cap T=\varnothing$. Write

$$
a \rho b \Leftrightarrow a, b \in I \text { or } a, b \in T \text {. }
$$

Then $\rho$ is a maximal modular congruence on $S$, where each element of $T$ is an identity for $\rho$ and $\rho$ is not cancellative.

Let $S$ be an inverse $H$-semigroup. For $e \in E$, let $T_{e}=\bigcup\left\{G_{f}: e \leqq f\right\}$. Define the relation $\rho^{(e)}$ on $S$ by

$$
a \rho^{(e)} b \Leftrightarrow a, b \in T_{e} \text { or } a, b \in T_{e}^{\prime}
$$

where $T_{e}^{\prime}=S-T_{e}$. If $e$ is not a minimum idempotent in $S$, we claim that $\rho^{(e)}$ is a maximal modular congruence on $S$ with identity $e$ and $\rho^{(e)}$ is not cancellative. Since $e \in T_{e}, T_{e} \neq \varnothing$. Let $d, b \in T_{e}$, say $d \in G_{f}, b \in G_{k}$. Then $e \leqq f$ and $e \leqq k$ imply $e \leqq f k$ so that $d b \in G_{f k} \subseteq T_{e}$ and $T_{e}$ is a subsemigroup of $S$. Assume $e$ is not minimum in $E$ so that $T_{e}^{\prime} \neq \varnothing$. Let $d \in T_{e}^{\prime}, b \in S$, say $d \in G_{f}$ and $b \in G_{k}$. Now $d \in T_{e}^{\prime}$ implies $f<e$ or $f ? e$. If $f<e$ then $f k<e$ and $d b \in G_{f k} \subseteq T_{e}^{\prime}$. If $f$ ? $e$ then $f k<e$ or $f k ? e$ and $d b \in T_{e}^{\prime}$. Thus $T_{e}^{\prime}$ is a right ideal of $S$. By Lemma $1.2, T_{e}^{\prime}$ is an ideal of $S$. It follows that $\rho^{(e)}$ is a maximal congruence on $S$ with identity $e$ and $\rho^{(e)}$ is not cancellative.

Let $\sigma$ be a maximal modular congruence on an inverse $H$-semigroup $S$. For each $e \in E$, let $H_{e}$ be the subgroup of $G_{e}$ induced by $\sigma$. Then, as in the proof of Theorem 2, we know that for $a, b \in S$, say $a \in G_{f}, b \in G_{k}, a \sigma b \Leftrightarrow a b^{-1} \in H_{f k}$ and $f \sigma(f k) \sigma k$. Let $a$ be an identity for $\sigma$, say $a \in G_{f}$. Then for each $s \in S$, (as) $\sigma s$ implies $(f a s) \sigma(f s)$ so that $(a s) \sigma(f s) \sigma s$. Thus $f$ is an identity for $\sigma$.

It is generally known that $\sigma$ is cancellative if and only if $E \subseteq \sigma_{e}$.

Suppose $\sigma$ is not cancellative and let $e \in E$ be an identity for $\sigma$. If $h \in E$ is an identity for $\sigma$, then $h \sigma(e h) \sigma e$ and $h \in \sigma_{e}$. Since $\sigma$ is not cancellative there exists $f \in E$ such that $f \notin \sigma_{e}$, so that $f$ is not an identity for $\sigma$. Let $I=\{f \in E: f$ is not an identity for $\sigma\}$. Then $I$ is an ideal in $E$. Let $J=\bigcup\left\{G_{f}: f \in I\right\}$. Then $J$ is an ideal of $S$ and $J^{\prime}$ is a semigroup of $S$. Oehmke [7] has shown that if $\sigma$ is a maximal congruence and $J$ any ideal of $S$, then either $J$ is contained in a $\sigma$-class $S_{0}$ (which is also an ideal of $S$ ) or $J$ contains an element of each $\sigma$-class. If $x \in \sigma_{e} \cap J$, then $x \sigma e$ and $x \in G_{f}$, for some $f \in I$. But then $x \sigma(e f)$ so that $e \sigma(e f)$ and, since also 
(ef) $\sigma f$, then $e \sigma f$ and $f \in I$, which is a contradiction. Hence $\sigma_{e} \cap J=\varnothing$ and there exists a $\sigma$-class $S_{0}$ such that $J \subseteq S_{0}$. Suppose there is some $b \in S_{0}$ such that $b \notin J$, that is, $b \in G_{h}$ where $h \sigma e$. Let $f \in I$. Then $b \sigma f$ implies $b f \in H_{h f}$ and $h \sigma(h f) \sigma f$, so that $f \sigma e$. Contradiction. Therefore $J=S_{0}$. Since $J$ is an ideal and $J^{\prime}$ is a semigroup, we have the maximal modular congruence $\sigma^{*}$ defined by $a \sigma^{*} b \Leftrightarrow a, b \in J$ or $a, b \in J^{\prime}$, where each element of $J$ is an identity for $\sigma^{*}$. Clearly $\sigma \leqq \sigma^{*}$. Hence $\sigma=\sigma^{*}$ and we have proved the following lemma.

LEMMA 3.1. If $\sigma$ is a maximal modular congruence on an inverse $H$-semigroup $S$, then $\sigma$ is cancellative or $\sigma$ has exactly two congruence classes, namely the semigroup of identities for $\sigma$ and the ideal of nonidentities for $\sigma$.

Suppose $\sigma$ is cancellative. Then $E \subseteq \sigma_{e}$ where $\sigma_{e}=\bigcup\left\{H_{f}: f \in E\right\}$. Since $\sigma$ is maximal, then $S / \sigma$ has no nontrivial congruences. Therefore $S / \sigma$ is the semigroup $\{0,1\}$ or $S / \sigma$ is simple. Since $\sigma$ is cancellative, it follows that $S / \sigma \neq\{0,1\}$. Since $S$ is an inverse $H$-semigroup, then $S / \sigma$ is an inverse $H$-semigroup. Therefore, by Lemma 1.2, every one-sided ideal of $S / \sigma$ is a two-sided ideal. Hence $S / \sigma$ is both left and right simple, so that $S / \sigma$ is a Hamiltonian group [1, p. 6]. Since $S / \sigma$ has no nontrivial congruences, then $S / \sigma$ has no nontrivial homomorphisms, so that $S / \sigma$ is a simple group. Since $S / \sigma$ is Hamiltonian, then $S / \sigma$ has no nontrivial subgroups. Hence $S / \sigma$ is a cyclic group of prime order. Thus there exists a prime number $p$ such that, for every $a \notin \sigma_{e}$, the $\sigma$-classes may be written as $\sigma_{a}, \sigma_{a^{2}}, \ldots, \sigma_{a^{p}}$, where $\sigma_{a^{p}}=\sigma_{e}$ and $a^{p}$ is an identity for $\sigma$. In fact, for every $c \in \sigma_{e}, c \sigma e$ implies $(c s) \sigma(e s) \sigma s$ and (sc) $\sigma(s e) \sigma s$ so that $c$ is an identity for $\sigma$. By a similar argument, if $a \notin \sigma_{e}$, then $a$ is not an identity for $\sigma$. If $G_{e}=H_{e}$ then $G_{e} \subseteq \sigma_{e}$. If $G_{e} \neq H_{e}$ then $\sigma$ partitions $G_{e}$ into cosets of $H_{e}$. The number of these cosets must be $p$, for otherwise $S / \sigma$ would contain a proper subgroup. Hence the cosets of $H_{e}$ must form a cyclic group of prime order and $H_{e}$ must be a maximal subgroup of $G_{e}$. We state these results in the following lemma:

LEMMA 3.2. If $\sigma$ is a maximal modular cancellative congruence on an inverse $H$-semigroup $S$, then $S / \sigma$ is a cyclic group of prime order $p$ such that for each nonidentity element $g$ for $\sigma$, the cosets of $S / \sigma$ are $\sigma_{e}, \sigma_{g}, \ldots, \sigma_{g^{p-1}}$. Moreover, if $\sigma^{\prime}$ is the restriction of $\sigma$ to $G_{e}$, then for each $e$ in $E, \sigma^{\prime}=\nu$ or $\sigma^{\prime}$ induces a maximal subgroup $H_{e}$ of $G_{e}$ where the cosets of $H_{e}$ form a cyclic group of prime order $p$.

LEMMA 3.3. If $T$ is a proper subsemigroup of $S$ such that for each $e \in E, T \cap G_{e}$ $=H_{e}$, where $H_{e}=G_{e}$ or $H_{e}$ is a maximal subgroup of index $p$ in $G_{e}$, and for each pair of groups $G_{e}, G_{f}$ in the semilattice $S$, where $e \leqq f$, the homomorphism $\varphi_{f, e}$ on $G_{f}$ into $G_{e}$ defined by $a \varphi_{f, e}=$ ae is a monomorphism, then $T$ induces a maximal modular cancellative congruence on $S$.

Proof. Define $\sigma$ on $S$ by $a \sigma b \Leftrightarrow a b^{-1} \in H_{k f}$, where $a \in G_{k}, b \in G_{f}$. Clearly, $\sigma$ is reflexive, symmetric and compatible. That $\sigma$ is transitive follows from the hypothe- 
sis that for each $e, f$ in $E$ where $f \geqq e$, the homomorphism $\varphi_{f, e}$ is a monomorphism. Therefore $\sigma$ is a congruence on $S$. It follows immediately from the definition of $\sigma$ that $T=\sigma_{e}$ and $\sigma$ is modular. Then $E \subseteq \sigma_{e}$ and $\sigma$ is cancellative. If $\sigma<\sigma^{\prime}$ where, for each $e \in E, \sigma^{\prime}$ induces the subgroup $K_{e}$ in $G_{e}$, then there exists $a, b \in S$ such that $a \sigma^{\prime} b$ and $a \phi b$. Say $a \in G_{f}, b \in G_{h}$. Then $a b^{-1} \in K_{f h}$ and $a b^{-1} \notin H_{f h}$ which implies that $H_{f h} \subset K_{f h}$ so that $K_{f h}=G_{f h}$, since $H_{f h}$ is then maximal in $G_{f h}$. If $k>f h$, then $G_{k}=K_{k}$. For $k<f h, H_{k} \subset\left(G_{f}\right) \varphi_{f h, k} \subseteq K_{k} \subseteq G_{k}$. Assume $K_{k} \subset G_{k}$. Since $H_{k}$ has index $p$ in $G_{k}$, then $K_{k}$ has finite index $j$ in $G_{k}$ and the index $m$ of $H_{k}$ in $K_{k}$ is such that $p=m j$ [4, p. 63]. Then either $m=p$ and $j=1$, or $m=1$ and $j=p$. If $m=1$, then $H_{k}=K_{k}$, which is a contradiction. If $j=1$, then $G_{k}=K_{k}$, contrary to the assumption. Thus, for $k<f h$ we must have $G_{k}=K_{k}$. If $k$ ? $f h$ then $f h k<f h$ and $G_{f h k}=K_{f h k}$. Since $\varphi_{k, f h k}$ is a monomorphism then $G_{k}=K_{k}$. Therefore $\sigma^{\prime}=\nu$ and $\sigma$ is maximal. This completes the proof.

Define the relation $\rho$ on $S$ as follows:

$$
x \rho y \Leftrightarrow \text { there exists } e \in E \text { such that } e x=e y .
$$

Clearly $\rho$ is a congruence on $S$. Then for each $e, f$ in $E, e \rho f$ since $(e f) e=e f=(e f) f$. Thus $E \subseteq \rho_{e}$. Once again we note that $S / \rho$ is an inverse $H$-semigroup containing exactly one idempotent so that $S / \rho$ is a Hamiltonian group. Let $\sigma$ be any maximal modular cancellative congruence on $S$ and let $a, b \in S$ such that $a \rho b$. Then

$$
\begin{aligned}
a \rho b & \Rightarrow \text { there exists } e \text { in } E \text { such that } e a=e b \\
& \Rightarrow(e a) \sigma(e b) \Rightarrow a \sigma b .
\end{aligned}
$$

Thus the intersection $\alpha$ of all the maximal modular cancellative congruences of $S$ is greater than or equal to $\rho$. Now for any $f, e$ in $E$, where $f<e$, if the homomorphism $\varphi_{e, f}$ is not a monomorphism then there exist $a \neq b$ in $G_{e}$ with $f a=f b$ so that $a \rho b \Rightarrow a \propto b$. Suppose that $S$ is $t$-semisimple. From Lemma 3.1, it is clear that the intersection $\beta$ of all the maximal modular noncancellative congruences of $S$ separates $S$ into its maximal subgroups. Thus, if $\varphi_{e, f}$ is not a monomorphism then $a \alpha b$ and $a \beta b$ imply $a \tau b$ so that $\tau \neq \iota$, contrary to the supposition. Let $a \neq e$, $a \in G_{e}, e \in E$. Since $a \beta e$ then there must be a maximal modular cancellative congruence $\sigma$ on $S$ such that $a \phi e$. From Lemma 3.2 it follows that there exists a maximal subgroup $H_{e}$ of index $p$ in $G_{e}$ such that $a \notin H_{e}$. Since we know that, for each $f \in E$, the restriction of $\sigma$ to $G_{f}$ induces a subgroup $H_{f}$ of $G_{f}$ such that $H_{f}$ is of index $p$ in $G_{f}$ or $H_{f}=G_{f}$, and since $E \subseteq \sigma_{e}$, then the union of these subgroups is a proper inverse subsemigroup of $S$. Let $\mathscr{T}$ be the collection of all inverse subsemigroups $T_{p}$ of $S$ such that, for each $e$ in $E, T_{p} \cap G_{e}=H_{e}$, where $H_{e}=G_{e}$ or $H_{e}$ is a maximal subgroup of prime index $p$ in $G_{e}$. Then we may say that, if $S$ is $t$-semisimple, then for each $e$ in $E$, for each $a \neq e$ in $G_{e}$, there exists $T_{p} \in \mathscr{T}$ such that $a \notin T_{p}$. Conversely, assume that for each $f, e$ in $E$, where $e<f, \varphi_{f, e}$ is a monomorphism; and for each $e$ in $E$, for each $a \neq e$ in $G_{e}$, there exists $T_{p} \in \mathscr{T}$ such that $a \in T_{p}$. Suppose $a \tau b$, where $a \in G_{e}, b \in G_{f}$. Since $\beta$ separates $S$ into its maximal 
subgroups, then $e=f$. If $a \neq b$ then $a b^{-1} \neq e$, and by assumption there exists $T_{p} \in \mathscr{T}$ such that $a b^{-1} \notin T_{p}$. By Lemma 3.3, it follows that there exists a maximal modular cancellative congruence $\sigma$ on $S$ separating $a$ and $b$. Thus if $a \tau b$, then $a=b$ and $S$ is $t$-semisimple. We can now state the main result of this section.

THEOREM 3. An inverse $H$-semigroup $S$ is $t$-semisimple if and only if for each pair of groups $G_{e}, G_{f}$ in the semilattice, with $f \geqq e$, the homomorphism $\varphi_{f, e}$ on $G_{f}$ into $G_{e}$, defined by $a \varphi_{f, e}=a e$, is a monomorphism, and for each $e$ in $E$, for each $a \neq e$ in $G_{e}$, there exists a subsemigroup $T_{p}$ of $S$ such that a $\notin T_{p}$ and, for each fin $E, T_{p} \cap G_{f}=H_{f}$, where $H_{f}=G_{f}$ or $H_{f}$ is a maximal subgroup of prime index $p$ in $G_{f}$.

COROLlaRY 3.1. $S$ is an inverse H-semigroup all of whose maximal modular congruences are cancellative if and only if $S$ is a Hamiltonian group.

COROLlary 3.2. $S$ is a t-semisimple inverse $H$-semigroup all of whose nontrivial maximal modular congruences are not cancellative if and only if $S$ is a semilattice.

COROLlaRY 3.3. If $S$ is a t-semisimple inverse H-semigroup, then $S$ is a semilattice of disjoint t-semisimple Hamiltonian groups.

Proof. Let $f \in E$. It suffices to show that $G_{f}$ is $t$-semisimple. Let $a, b$ be distinct elements of $G_{f}$. Since $a \beta b$ then there must be a maximal modular cancellative congruence $\sigma$ on $S$ such that $a \not b$. Let the $\sigma$-classes be $\sigma_{e}, \sigma_{g}, \ldots, \sigma_{g}^{p-1}$. Let $\sigma^{\prime}$ be the restriction of $\sigma$ to $G_{f}$. Then $a \notin b$. Now $a \sigma b$ implies either $a \notin \sigma_{e}$ or $b \notin \sigma_{e}$. Say $a \notin \sigma_{e}$. Then the $\sigma$-classes may be written as $\sigma_{f}, \sigma_{a}, \ldots, \sigma_{a^{p-1}}$ so that the $\sigma^{\prime}-$ classes are $\sigma_{f}^{\prime}, \sigma_{a}^{\prime}, \ldots, \sigma_{a^{p-1}}^{\prime}$. Further, $G_{f} / \sigma^{\prime}$ is a cyclic group of prime order so that $\sigma^{\prime}$ is a maximal congruence on $G_{f}$ separating $a$ and $b$. Hence $G_{f}$ is $t$-semisimple.

Lemma 3.4.1. A t-semisimple Hamiltonian group is commutative.

Proof. Let $G$ be a $t$-semisimple Hamiltonian group and assume $G$ is not commutative. Then $G=Q \times A \times B$ where $Q$ is a quaternion group, $A$ a commutative group of exponent two, $B$ a commutative group where each element has odd order. Since $Q$ is a finite $p$-group then $Q$ is nilpotent, and since $A$ and $B$ are commutative then $A$ and $B$ are nilpotent [3, p. 155, p. 149]. Therefore $G$ is nilpotent [5, p. 212]. Now every maximal subgroup of a nilpotent group is normal, is of prime index, and contains the derived group [3, p. 154]. Hence the intersection $\Phi$ of the maximal (normal) subgroups of $G$ contains the derived group $G^{\prime}$. But $G$ is $t$-semisimple so that its Frattini subgroup $\Phi$ consists of the identity only. Thus $G^{\prime}$ contains the identity only and $G$ is commutative. But this contradicts our assumption so that the result follows.

Corollary 3.4. If $S$ is a t-semisimple inverse H-semigroup, then $S$ is commutative.

COROLlary 3.5. If $S$ is an inverse $H$-semigroup with a minimum idempotent $e$, then $S$ is $t$-semisimple if and only if $G_{e}$ is $t$-semisimple and, for each group $G_{f}$ in the semilattice with $f \geqq e$, the homomorphism $\varphi_{f, e}$ on $G_{f}$ into $G_{e}$, defined by a $\varphi_{f, e}$, is a monomorphism. 
Proof. Only the sufficiency requires proof. Let $f, h \in E$ with $f \geqq h$ and assume there exist $a \neq b$ in $G_{f}$ such that $a h=b h$ in $G_{h}$. Then $a e=a h e=b h e=b e$ in $G_{e}$ implies $a=b$, since $\varphi_{f, e}$ is a monomorphism. Contradiction. Hence, for each $f, h$ in $E$, where $f \geqq h, \varphi_{f, h}$ is a monomorphism. Let $f \in E$ and $a \neq f$ in $G_{f}$. Assume $a \in \Phi_{f}$. Since $\varphi_{f, e}$ is injective then $a e \neq e$ in $G_{e}$, so that there exists a maximal subgroup $H_{e}$ in $G_{e}$ such that $a e \notin H_{e}$. Thus $a=a e \varphi_{f, e}^{-1} \notin H_{e} \varphi_{f, e}^{-1}=H_{f}$ in $G_{f}$. But $H_{f}$ is a maximal subgroup of $G_{f}$ and $a \notin H_{f}$ imply $a \notin \Phi_{f}$. Contradiction. Hence $G_{f}$ is $t$-semisimple. It remains to show that for each $f$ in $E$, for each $a \neq f$ in $G_{f}$, there exists a subsemigroup $T_{p}$ of $S$ such that $a \notin T_{p}$ and, for each $h$ in $E, T_{p} \cap G_{f}=H_{h}$, where $H_{h}=G_{h}$ or $H_{h}$ is a maximal subgroup of prime index $p$ in $G_{h}$. Let $a \neq f$ in $G_{f}$. Since $G_{f}$ is $t$-semisimple, there exists a maximal subgroup $H_{f}$ in $G_{f}$ such that $a \notin H_{f}$. It follows that $G_{f} / H_{f}$ has no nontrivial subgroups and is therefore cyclic of prime order $p$ so that the cosets of $H_{f}$ may be written as $H_{f}, H_{f} a, \ldots, H_{f} a^{p-1} . H_{f} e$ is a subgroup of $G_{e}$ which does not contain $e a$. Let $H_{e}$ be a subgroup of $G_{e}$ maximal with respect to not containing ea and such that $H_{f} e \subseteq H_{e}[8, \mathrm{p} .22]$. But then $H_{e}$ is a maximal subgroup of $G_{e}$ and $G_{e} / H_{e}$ is cyclic of prime order. Since $\varphi_{f, e}$ is a monomorphism from $G_{f}$ into $G_{e}$ and $H_{f}$ is a maximal subgroup of $G_{f}$, it follows that $e a^{i} \notin H_{e}$, $1 \leqq i \leqq p-1$, and $H_{e}, H_{e} a, \ldots, H_{e} a^{p-1}$ are distinct cosets of $H_{e}$. Hence these must be all the cosets of $H_{e}$ so that $H_{e}$ is maximal and of index $p$ in $G_{e}$. For each $h$ in $E$, let $H_{h}=\left(H_{e}\right) \varphi_{h, e}$. It follows that for each $H_{h}$ either $H_{h}=G_{h}$ or $H_{h}$ is maximal and of index $p$ in $G_{h}$. Further, for $h, k$ in $E$, let $x \in H_{h}, y \in H_{k}$. Then

$$
x e, y e \in H_{e} \Rightarrow x y e \in H_{e} \Rightarrow x y \in H_{h k} .
$$

Thus the union of all $H_{h}, h \in E$, as defined above, is a subsemigroup $T_{p}$ with the desired properties and the proof is complete.

COROllary 3.6. If $S$ is a finite inverse $H$-semigroup, then $S$ is $t$-semisimple if and only if $G_{e}$ is t-semisimple, where $e$ is the minimum idempotent of $E$, and for each subgroup $G_{f}, f>e$, the homomorphism $\varphi_{f, e}$ from $G_{f}$ into $G_{e}$, defined by $a \varphi_{f, e}=a e$, is a monomorphism.

COROLlARY 3.7. If $S$ is a t-semisimple inverse H-semigroup with no nontrivial modular congruences, then $S$ is either a cyclic group of prime order or the unique semilattice of two elements.

Proof. Since $S$ is $t$-semisimple, it has maximal modular congruences, that is, $\iota$ is a maximal modular congruence. Since there is no nontrivial modular (noncancellative) congruence on $S$, then $S$ is a group or $S$ is the semilattice of two elements. In the former case, any congruence on $S$ would be modular, so it follows that $S$ has no nontrivial subgroups, hence is cyclic of prime order.

COROLlaRY 3.8. If $S$ is an inverse H-semigroup with zero, then $S$ is t-semisimple if and only if $S$ is a semilattice. 


\section{REFERENCES}

1. A. H. Clifford and G. B. Preston, Algebraic theory of semigroups. Vol. I, Math. Surveys, no. 7, Amer. Math. Soc., Providence, R. I., 1961. MR 24 \#A2627.

2. - Algebraic theory of semigroups. Vol. II, Math. Surveys, no. 7, Amer. Math. Soc., Providence, R. I., 1967. MR 36 \#1558.

3. Marshall Hall, Jr., The theory of groups, Macmillan, New York, 1959. MR 21 \#1996.

4. A. G. Kuroš, Theory of groups, 2nd ed., GITTL, Moscow, 1953; English transl., Vol. 1, Chelsea, New York, 1955. MR 15, 501; MR 17, 124.

5. — Theory of groups, GITTL, Moscow, 1953; English transl., Vol. 2, Chelsea, New York, 1956. MR 15, 501; MR 18, 188.

6. R. H. Oehmke, A generalization of commutativity for semigroups, Portugal. Math. 24 (1965), 179-187. MR 35 \#5529.

7. _- On maximal congruences and finite semisimple semigroups, Trans. Amer. Math. Soc. 125 (1966), 223-237. MR 34 \#2739.

8. H. J. Zassenhaus, The theory of groups, 2nd ed., Chelsea, New York, 1958. MR 19, 939.

Department of Mathematics, Seton Hill College, Greensburg, Pennsylvania 15601 